\title{
Advantages of Large Area SDD Detectors for Analysis in Analytical TEMs.
}

\author{
N. Rowlands*, S. Yamaguchi** and M. Ishida**. \\ * Oxford Instruments NanoAnalysis, 300 Baker Avenue, Suite 150, Concord, Massachusetts, USA \\ ** Oxford Instruments KK, Tokyo, Japan
}

Silicon drift detectors have become the detector of choice for SEM-based EDS systems, taking the place of the traditional $\mathrm{Si}(\mathrm{Li})$ detectors. The acknowledged advantages that SDDs have over $\mathrm{Si}(\mathrm{Li})$ detectors include liquid nitrogen $\left(\mathrm{LN}_{2}\right)$ free operation and the capability to rapidly process large count rates without much sacrifice of spectral resolution. When combined with new pulse processor technology these systems are very stable and can give highly accurate quantitative data over a wide range of count rates. A new generation of large area SDDs are now available for SEMs. These detectors combine the advantages of SDD with large solid angle to maximise the X-ray count rate collected at any given condition. These detectors have also been used as STEM detectors in the SEM where very small particulates suspended on thin films may now be analyzed - a process that until recently required the use of a TEM [1].

With the introduction of a TEM version of the X-Max $80 \mathrm{~mm} 2$ detector, large area SDD detectors are now also available for the TEM. The increased solid angle of these detectors is highly beneficial in improving collection efficiency, for example with $\mathrm{LaB}_{6}$ or W TEMs when generating nano-probes with low beam currents for the analysis of nano-structures. This increased solid angle also makes these detectors useful for the analysis of polymers and biological materials where high beam currents may damage the sample. The ability of SDDs to handle large count rates is particularly advantageous for data collection in modern field-emission, aberration corrected TEMs where nano-probes with extremely high beam currents can generate very large X-ray count rates. It is also useful in situations where high X-Ray intensity is generated when analyzing thicker sections as used in the study of microstructure of engineering samples [2] or where samples contain heavy metals such as tungsten or gold.

Although the high energy TEM environment is harsh for an X-ray detector, tests at a number of installations have confirmed that the excellent spectral performance of large area SDD can still be achieved in a TEM. For example, Fig. 1. is a typical spectrum collected from a Mn film, which shows excellent peak resolution and peak to background ratios. These detectors also show excellent low energy sensitivity, for example Fig. 2. shows the detection of the very low energy SiLl peak at $90 \mathrm{eV}$ in a spectrum collected from a silicon film.

The higher count rates now enable fast X-ray mapping on the TEM, with nano-structures being easily resolved in much shorter times than were previously possible. In addition to acquiring X-Ray spectrum image datacubes in much shorter times, there is enough data to justify processing these datasets beyond the normal display of energy-window integral digital maps. For example Fig. 3 shows quantitative maps that were calculated from a SmartMap spectrum image collected in 30 minutes and include full spectrum processing at each pixel. These images demonstrate the ability to correctly display element distribution where peaks overlap, as is the case for the Si K and W M lines. It also shows the capability to investigate the distribution of oxide nanostructures in semiconductors. 
The same capabilities that have driven the rapid take up of large area SDDs on SEM are just as relevant for TEM. Practical experience is showing that large improvements in EDS performance can be achieved on the TEM in practice and indicate that a switch to SDD for TEM is also likely.

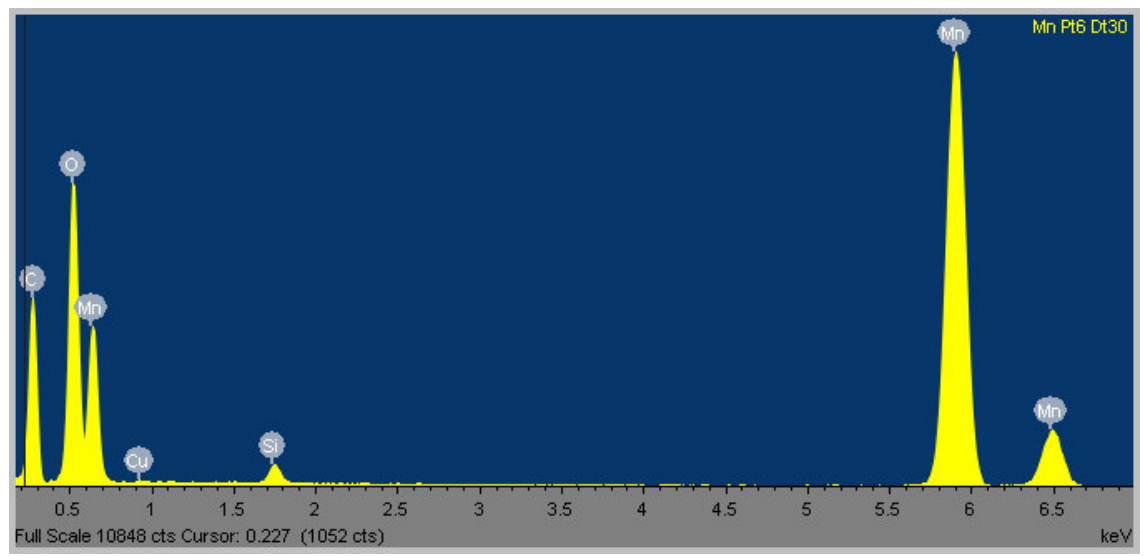

Figure 1. Spectrum collected from a manganese sample using $200 \mathrm{kV}$ on a JEOL JEM $2100 \mathrm{LaB}_{6}$ STEM. The spectrum shows excellent peak to background and a resolution at $\mathrm{Mn}$ of $126 \mathrm{eV}$.

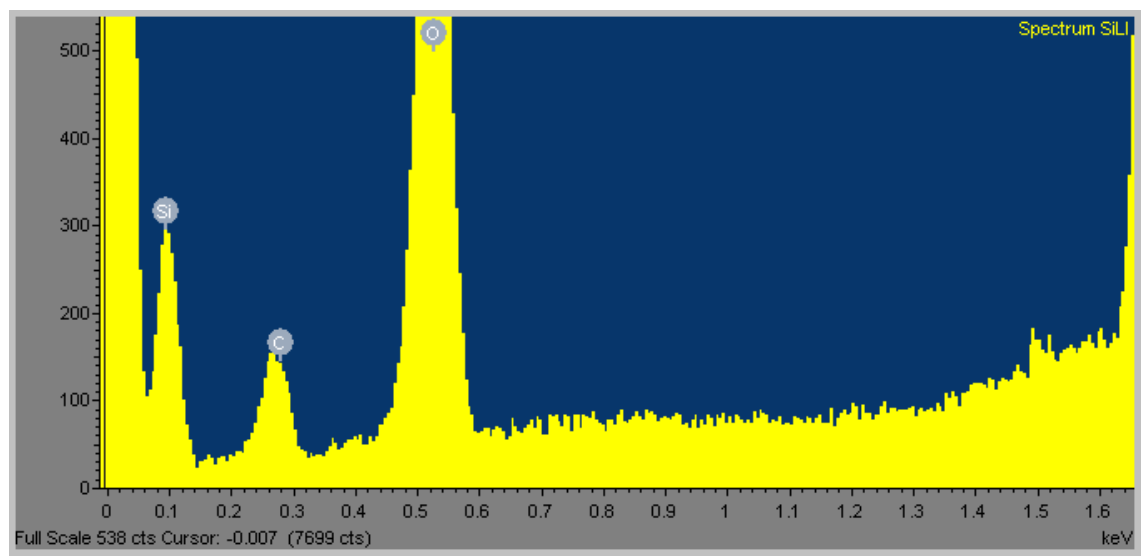

Figure 2. Spectrum collected from a silicon sample using $200 \mathrm{kV}$ on a JEOL JEM $2100 \mathrm{LaB}_{6}$ STEM. The spectrum shows detection of the SiLl line at $90 \mathrm{eV}$.

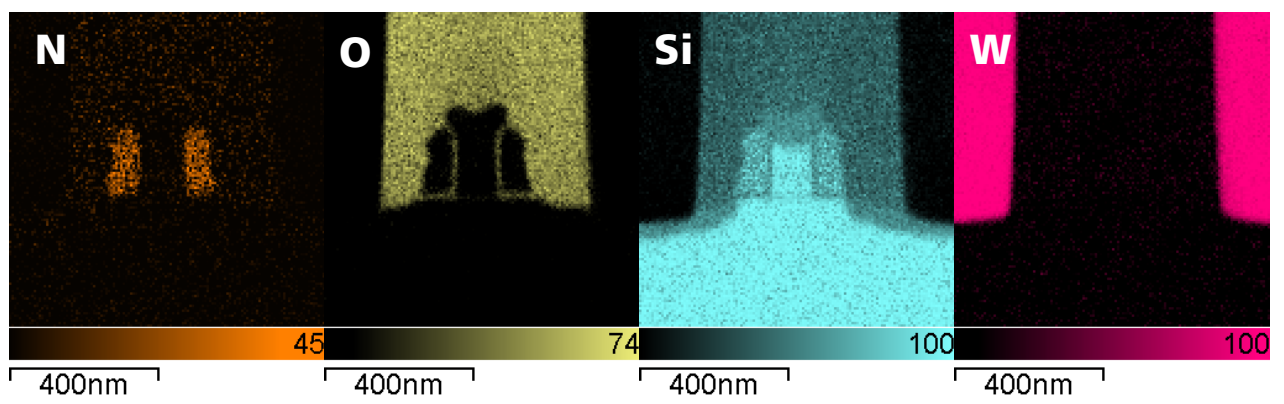

Figure 3. X-Ray quantitative maps from a semi-conductor sample demonstrate the ability to show nano structures and the deconvolution of the overlapping Si K peaks from the W M peaks

[1] N. Rowlands, Microscopy and Microanalysis (2009), 15:548-549.

[2] M. Chu, Microscopy and Analysis (2009), In Press 